\title{
Emma Álvarez-Prendes Polyfonctionnalité adverbiale, grammaticalisation et subjectivation : le cas de sérieusement, seriamente et en serio
}

https://doi.org/10.1515/zrp-2018-0028

\begin{abstract}
In this paper we analyze the contemporary uses of three equivalent adverbs in French and Spanish (sérieusement, seriamente and en serio) in order to characterize them by some salient linguistic properties. We will also establish the emergence of these uses in the history of the French and Spanish languages and try to determine the historical links between them. To achieve these goals we have created a personal corpus with more than 200 occurrences of each adverb from very diverse types of texts (novels, journals, Internet, oral, data bases such as Frantext, Corpus Le Monde Diplomatique, Corpus de Referencia del Español Actual and Corpus Diacrónico del Español, etc.). Our main hypothesis is that those three adverbs are the result of a similar complex process of grammaticalization.
\end{abstract}

Keywords: adverbs, polyfunctionality, grammaticalization, subjectivation, Fr. sérieusement, Sp. seriamente, Sp. en serio

Mots clés: polyfonctionnalité, grammaticalisation, subjectivation, fr. sérieusement, esp. seriamente, esp. en serio

\section{Introduction}

Cette étude fait suite à deux articles précédents (Álvarez-Prendes, 2016 et ÁlvarezPrendes, soumis) dans lesquels nous avons étudié les emplois et les propriétés de l'adverbe sérieusement, et le fonctionnement et l'évolution historique des adverbes honnêtement (français) et honestamente (espagnol), respectivement. Le travail présent vise à élucider le fonctionnement des adverbes sérieusement (français) et seriamente et en serio (espagnol), ainsi qu'à établir les liens historiques entre leurs différents emplois à l'aide de la notion de subjectivation de Traugott (1995). Nous

Adresse de correspondance : Prof. Dr. Emma Álvarez-Prendes, Universidad de Oviedo, Facultad de Filosofía y Letras, Calle Amparo Pedregal, s/n, E-33011-Oviedo,

E-Mail : alvarezemma@uniovi.es 
avons choisi une autre langue romane (à savoir l'espagnol) afin de pouvoir élargir la recherche déjà effectuée sur la langue française et de comparer ainsi les régularités repérées dans deux langues proches et néanmoins différentes.

Notre hypothèse de départ est que le passage de l'emploi initial en tant qu'adverbe de manière orienté vers le sujet ${ }^{1}$ à l'emploi comme adverbe de manière quantifieur intensif et, plus tard, à l'emploi comme adverbe disjonctif de style est l'aboutissement d'un processus de subjectivation dans le sens de Traugott, et ceci aussi bien en français qu'en espagnol.

Les données examinées ont été tirées d'un corpus personnel de plus de deux centaines d'occurrences de chaque adverbe d'origine variée (textes littéraires, journalistiques, Internet, oral, bases de données Frantext, Le Monde et Corpus de Referencia del Español Actual [CREA], Corpus Diacrónico del Español [CORDE], etc.). Nous avons soumis ces occurrences à des tests linguistiques (tests empruntés pour la plupart à Schlyter 1977 et Molinier/Levrier 2000) afin de déceler des patterns dans leur fonctionnement. Nous avons aussi eu recours à deux exemples forgés (l'un pour le français, l'autre pour l'espagnol) dans des cas où notre corpus ne fournissait pas d'exemple satisfaisant et où nous avions la certitude, corroborée par des informateurs natifs, que ce genre d'exemples pouvait exister dans les deux langues mentionnées (cf. exemples 13 et 15).

Ce travail s'inscrit dans la lignée des travaux du groupe OPÉRAS sur les opérateurs sémantico-pragmatiques de la langue française (c'est-à-dire, « des entités linguistiques dont l'emploi a pour effet de mettre en place une stratégie discursive spécifique » et "dont le fonctionnement peut être appréhendé à travers des propriétés morphosyntaxiques, sémantiques et pragmatiques distinctes », cf. Anscombre/Donaire/Haillet 2013). Les adverbes - dont sérieusement, seriamente et en serio - peuvent être rangés dans cette catégorie plus large des opérateurs sémantico-pragmatiques.

\section{Analyse linguistique de sérieusement, seriamente et en serio : emplois et propriétés}

Après avoir soumis les exemples de notre corpus à des tests de type syntaxique et sémantique (i.e. extraction par c'est... que, possibilité à figurer en tête de phrase négative, possibilité d'être le focus de la négation, diverses possibilités de para-

1 Sauf indication du contraire, nous aurons recours dans cet article à la terminologie et à la classification des adverbes en -ment établie par Molinier/Levrier (2000). 
phrase, enchaînements, etc.), nous avons identifié trois emplois des adverbes sérieusement, seriamente et en serio :

- Un adverbe de manière orienté vers le sujet, qui caractérise la réalisation du procès évoqué par le verbe de l'énoncé (Exemples : « Alexandre la considéra sérieusement »; " Australia es muy grande, tiene un tamaño muy superior al de las islas Canarias y, sin embargo, los australianos se plantean seriamente erradicar los gatos de su mundo »; " Por vez primera me acercaba en serio al dominio de las ciencias de la naturaleza »).

- Un adverbe de manière quantifieur intensif, qui indique un degré moyen ou un peu plus élevé que la moyenne du contenu de l'adjectif ou du verbe qu'il modifie (Exemples : «Il n'était pas surprenant de voir l'hémicycle sérieusement clairsemé lors de la séance de lundi soir »; "Grandes extensiones de bosques centroeuropeos, especialmente de coníferas, están seriamente afectadas por esta contaminación »).

- Un adverbe disjonctif de style, qui montre l'attitude du locuteur vis-à-vis de son propre dire (Exemples : « Je flambe ! Sérieusement, je dois récupérer la quarantaine de Chinois qui se sont échappés dans votre casino, pour leur faire boire du vin et caresser des vaches »; " Mientras no cambies, en serio, ni serás feliz, ni conseguirás la paz que anhelas »).

Comme nous venons de le constater, l'adverbe sérieusement est bien le seul à connaitre trois emplois différents (adverbe de manière orienté vers le sujet, adverbe de manière quantifieur intensif et adverbe disjonctif de style), tandis que les adverbes espagnols ne connaissent, eux, que deux emplois chacun : adverbe de manière orienté vers le sujet et adverbe de manière quantifieur intensif pour seriamente, et adverbe de manière orienté vers le sujet et adverbe disjonctif de style pour en serio.

Nous pouvons remarquer que tous trois partagent l'emploi comme adverbe de manière orienté vers le sujet. En revanche, seulement sérieusement et seriamente peuvent fonctionner comme un adverbe de manière quantifieur, et seulement sérieusement et en serio comme un adverbe disjonctif de style. Posé en d'autres termes, l'adverbe français possède deux emplois intraprédicatifs (Guimier 1996) et un emploi exophrastique, alors que les adverbes espagnols possèdent soit deux emplois intraprédicatifs (seriamente), soit un emploi intraprédicatif et un emploi exophrastique (en serio).

Nous avons résumé ces résultats dans le tableau suivant (cf. tableau 1) : 
Tableau 1

\begin{tabular}{llll}
\hline Emploi / Adverbe & sérieusement & seriamente & en serio \\
\hline Adverbe de manière orienté vers le sujet $^{\text {sérieusement }}{ }_{1}$ & seriamente $_{1}$ & en serio $_{1}$ \\
\hline Adverbe de manière quantifieur intensif & sérieusement $_{2}$ & seriamente $_{2}$ & - \\
\hline Adverbe disjonctif de style & sérieusement $_{3}$ & - & en serio $_{2}$ \\
\hline
\end{tabular}

Illustrons le premier fonctionnement mentionné - celui en tant qu'adverbe de manière orienté vers le sujet - à l'aide d'exemples de notre corpus :

(1) Tu parles sérieusement? (Gérard Delteil, Gombo).

(2) Alexandre la considéra sérieusement (Katherine Pancol, Les yeux jaunes des crocodiles).

(3) Marc, qui n'était pas de mauvaise foi, réfléchit sérieusement à cette phrase (Fred Vargas, Debout les morts).

(4) Trois quarts d'heure plus tard, j’ai fini de lire Le Canard enchaîné, bouclé le dernier Douglas Kennedy et je songe sérieusement à décliner mon identité afin de faire accélérer les choses (Zoé Shepard, Absolument débordée).

(5) Australia es muy grande, tiene un tamaño muy superior al de las islas Canarias y, sin embargo, los australianos se plantean seriamente erradicar los gatos de su mundo (CREA).

(6) Los grupos enemigos y sus jefes suelen ser percibidos como tan perversos y mal intencionados que no vale la pena negociar seriamente con ellos, o bien se les cree tan débiles o estúpidos que se considera que todo esfuerzo para conciliar las diferencias estará destinado al fracaso (CREA).

(7) A principios de la década de los años 60, diversos organismos internacionales empezaron a preocuparse seriamente del asunto y la FAO (Organización de las Naciones Unidas para la Agricultura y la Alimentación) convocó una reunión de la cual surgió un Cuadro de Expertos en Prospección de Plantas (CREA).

(8) Hace unos años, Margulis tuvo la feliz idea de plantear seriamente que uno de los motores de la evolución es la simbiosis entre especies que se encuentran en contacto. (CREA).

(9) Los economistas liberales pronto le [sic] tomaron en serio. (CREA).

(10) Por vez primera me acercaba en serio al dominio de las ciencias de la naturaleza.

(11) Y vivirás en serio el catolicismo (CREA).

(12) Unos se entregarán, otros se tomarán en serio su vida interior (CREA).

Dans ces exemples les adverbes sérieusement (exemples 1-4), seriamente (exemples 5-8) et en serio (9-12) caractérisent toujours la réalisation du procès évoqué par le verbe de l'énoncé. En tant qu'adverbes intégrés à la proposition, l'énoncé dans lequel ils s'inscrivent présente une structure de surface $S(X)$, où $S$ représente l'adverbe et $X$ le segment matériel qu'il accompagne, l'adverbe étant habituellement postposé au verbe sur lequel il porte. 
Cet usage répond aux deux conditions proposées par Molinier $(1990,29)$ pour les adverbes intégrés à la proposition : l'adverbe peut être extrait dans une phrase clivée (propriété uniquement possible en langue française : C'est sérieusement que tu parles ?) et il ne peut pas être détachée en tête de phrase négative.

Par ailleurs, dans cet emploi l'adverbe peut être foyer de la négation (i.e. $\mathrm{Tu}$ parles mais tu ne parles pas sérieusement; Los australianos se plantean erradicar los gatos de su mundo, pero no se lo plantean seriamente; Por vez primera me acercaba al dominio de las ciencias de la naturaleza, pero no me acercaba en serio) et constituer à lui seul une réponse (cf. exemples 13-15) :

(13) Comment s'est-il comporté ? Sérieusement (exemple forgé).

(14) - ¿ Llegó a plantearse seriamente encabezar la lista ?

- Sí. Seriamente. Era mi obligación reflexionarlo en profundidad, y lo hice.

(<http://javierfernandez.es/noticias/me-planteeacute-seriamente-ser-candidatopero_69.html\#sthash.Td8GVplm.dpuf> [dérniere consultation : 14.09.2017])

(15) ¿ Cómo se debe tomar el asunto ? En serio (exemple forgé).

En ce qui concerne les propriétés sémantiques, dans cet usage sérieusement, seriamente et en serio modifient le verbe de l'énoncé, d'où les paraphrases possibles par de (d'une) manière / façon + Adj en français (i.e. Tu parles de façon sérieuse) ou par de manera / forma + Adj en espagnol (i.e. Los australianos se plantean de manera seria erradicar los gatos de su mundo ; Por vez primera me acercaba de manera seria al dominio de las ciencias de la naturaleza).

$\mathrm{Au}$ cours de notre recherche nous sommes tombées sur des occurrences d'adverbes intégrés à la proposition qui ne répondaient pas vraiment aux caractéristiques de l'adverbe de manière orienté vers le sujet que nous venons d'exposer. Après avoir soumis ces nouvelles occurrences à des tests syntaxiques et sémantiques, nous avons identifié un deuxième emploi de ces adverbes : celui en tant qu'adverbe de manière quantifieur intensif. Seulement les adverbes sérieusement et seriamente acceptent cet usage. En voyons ci-après quelques exemples :

(16) Le PSG énerve sérieusement Guy Roux (<www.actufoot.net> [dérniere consultation : 14.09.2017]).

(17) Il n'était pas surprenant de voir l'hémicycle sérieusement clairsemé lors de la séance de lundi soir (Le Monde, 29/10/2013).

(18) Oui. La voiture rouge avait changé de place. Alexandra a dû rétracter sa première déclaration, elle s'est fait sérieusement engueuler et a avoué s'être absentée de onze heures un quart à trois heures du matin (Fred Vargas, Debout les morts).

(19) Il paraît pourtant qu'il a sérieusement importuné Noémie Labarrière (Brigitte Aubert, Funérarium).

(20) Grandes extensiones de bosques centroeuropeos, especialmente de coníferas, están seriamente afectadas por esta contaminación (CREA). 
(21) En Escandinavia, por ejemplo, se afirma que el bosque boreal se encuentra seriamente amenazado por las precipitaciones ácidas y que las pesquerías se encuentran asimismo afectadas por los contaminantes atmosféricos (CREA).

(22) Además de estar seriamente enferma, había recibido un tiro en la espalda (CREA).

(23) En la actualidad este modelo lineal de la innovación tecnológica está seriamente cuestionado (CREA).

Dans cet usage, les adverbes sérieusement (exemples 16-19) et seriamente (exemples 20-23) indiquent un degré moyen ou un peu plus élevé que la moyenne du contenu de l'adjectif ou du verbe qu'ils modifient. Étant donné que nous sommes face à des adverbes intégrés à la proposition, ils ne peuvent pas être détachés en tête de phrase négative et la structure de surface des énoncés dans lesquels ils figurent est $S(X)$, où $S$ représente l'adverbe et $X$ le segment matériel qu'il accompagne, l'adverbe se situant normalement en antéposition vis-à-vis du verbe ou de l'adjectif sur lequel il porte. Notons à ce sujet que la place de l'adverbe dans l'énoncé reste un élément crucial pour déterminer sa valeur : postposé, on aura tendance à en faire une lecture en tant qu'adverbe de manière orienté vers le sujet (donc sérieusement / $_{1}$ seriamente $_{1}$ ) ; antéposé, nous pencherons pour l'interprétation comme adverbe de manière quantifieur intensif (donc sérieusement $t_{2} /$ seria- $^{-}$ mente $_{2}$ ). Comparons à cet égard les deux exemples suivants : Il l'a devancé sérieusement dans la course (= il l'a devancé sans rire, sans se moquer) vs. Il l'a sérieusement devancé dans la course (= il l'a beaucoup devancé).

En outre, sérieusement et seriamente font tous deux partie des adverbes de manière quantifieurs intensifs qui peuvent être focus de la négation (i.e. L'hémicycle était clairsemé, mais il n'était pas sérieusement clairsemé ; Grandes extensiones de bosques centroeuropeos, especialmente de coníferas, están afectadas por esta contaminación, pero no están seriamente afectadas). D'un point de vue sémantique, sérieusement et seriamente peuvent être considérés comme des équivalents, plus nuancés, d'adverbes comme très, beaucoup, muy, mucho, etc. (Schlyter 1977) ; d'où le fait que ni sérieusement ni seriamente ne puissent être coordonnés dans le même énoncé avec des adverbes purement d'intensité. D'autre part, ils admettront comme paraphrase la paraphrase par Le $N$ est Adj / El $N$ es Adj (i.e. L'énervement de Guy Roux est sérieux ; La contaminación es seria).

Une dernière caractéristique de cet emploi est que sérieusement et seriamente portent toujours sur des verbes ou des adjectifs dont découlerait une conséquence plutôt négative si on dépassait un certain degré ou une certaine intensité (i.e. énerver, importuner, ébranler, chauffer, clairsemé, etc. ; afectar, amenazar, enfermar, cuestionar, etc.).

Il nous reste encore à examiner un troisième fonctionnement de ces adverbes : l'usage comme adverbe disjonctif de style ou, plus précisément, comme 
adverbe d'énonciation (Molinier 1990), ou encore à l'intérieur de cette dernière sous-classe, comme adverbe qui concerne la disposition psychologique ou morale du locuteur vis-à-vis de l'interlocuteur (Molinier 2009, 11). Regardons quelques exemples de sérieusement et en serio dans cet emploi :

(24) Je flambe ! Sérieusement, je dois récupérer la quarantaine de Chinois qui se sont échappés dans votre casino, pour leur faire boire du vin et caresser des vaches (Zoé Shepard, Absolument débordée).

(25) Non... Sérieusement, d'après nos constatations, vers les cinq heures du mat', reprit-il, tout ce petit monde est fatigué après une longue nuit de labeur. Alors ça roupille, la vigilance se relâche. C'est le moment idéal pour la percée (Thierry Jonquet, Ils sont votre épouvante et vous êtes leur crainte).

(26) En Jamaïque, «tu ne peux rien acheter avec 1 dollar, sérieusement », s'exclame sur Twitter Laura Edwards (Le Monde, 19/10/2013).

(27) Rempart contre l'islamisme, sérieusement, le régime qui a, pendant presque quinze ans, hébergé le bureau politique du Hamas, branche palestinienne des Frères musulmans et fer de lance, à ce titre, d'une des formes les plus radicales du fondamentalisme ? Rempart contre l'islamisme ? (Bernard Henry-Levy, Le Monde, 02/09/2013).

(28) Mientras no cambies, en serio, ni serás feliz, ni conseguirás la paz que anhelas (CREA).

(29) Bueno, bueno, ahora en serio - puntualiza una tercera- : está quedando pero... (CREA).

(30) Ya más en serio, no estaría mal que recuperáramos ese verdadero... (CREA).

(31) Bueno bueno, pero por circunstancias que no vienen al caso. Eso. Y y además yo estaba enfermo. Ya. Estaba enfermo y, bueno, los otros no quieras saber, los otros compañeros. Sí, sí. Estaba malísimo, en serio. Ya, sí hombre, ya. Malísimo (CREA).

Dans cet usage, sérieusement (exemples 24-27) et en serio (exemples 28-31) montrent l'attitude du locuteur vis-à-vis de son propre dire. En tant qu'adverbes de phrase, ils remplissent les deux conditions proposées par Molinier pour identifier ce type d'adverbes : ils peuvent figurer en position détachée en tête de phrase négative (i.e. Sérieusement, je ne dois pas récupérer la quarantaine de Chinois qui se sont échappés dans votre casino; En serio, ni serás feliz, ni conseguirás la paz que anhelas) et ne peuvent pas être extraits par une clivée. Ces deux conditions confirment qu'ils opèrent toujours en dehors du cadre propositionnel et qu'ils sont indépendants du contenu de la proposition. En tant qu'adverbes de phrase, leur structure de surface est $S(X, Y)$, où S représente l'adverbe et $\mathrm{X}$ et $\mathrm{Y}$, les deux segments matériels qu'il accompagne; dans sa version canonique cette structure de surface prendra une forme $: X$ sérieusement $_{3} /$ en serio $_{2} Y$.

Comme adverbes d'énonciation, sérieusement, seriamente et en serio répondent également aux conditions suivantes : ils se trouvent toujours en position détachée et n’ont aucune restriction sélectionnelle vis-à-vis de l'énoncé qu'ils accompagnent (Rodríguez Ramalle 2003). 
Outre ces deux conditions communes à la classe des adverbes d'énonciation dans son ensemble, sérieusement et en serio présentent dans cet emploi deux caractéristiques qui résultent de leur indépendance du contenu de l'énoncé : ils ne peuvent pas être focus de la négation et peuvent apparaître en tête d'une phrase déclarative affirmative (cf. exemples 24 et 32) ou négative (cf. exemples 33 et 34), interrogative (cf. exemples 35 et 36) et même impérative (cf. exemples 37 et 38) :

(32) En serio - puntualiza una tercera- : está quedando pero... (CREA).

(33) Sérieusement, en Jamaïque tu ne peux rien acheter avec 1 dollar (Le Monde, 19/10/ 2013).

(34) En serio, ni serás feliz, ni conseguirás la paz que anhelas (CREA).

(35) Sérieusement, rempart contre l'islamisme le régime qui a, pendant presque quinze ans, hébergé le bureau politique du Hamas, branche palestinienne des Frères musulmans et fer de lance, à ce titre, d'une des formes les plus radicales du fondamentalisme ? (Bernard Henry-Levy, Le Monde, 02/09/2013).

(36) $¿$ En serio, vas a ir ? (CREA).

(37) Sérieusement, laisse tomber (oral).

(38) En serio, olvídalo (CREA).

Quant à leur distribution, dans cet usage sérieusement et en serio figurent surtout en position initiale détachée (cf. exemples 24-25 et 32) ou en position insérée (cf. exemples 27 et 28-30), mais peuvent également figurer en position finale détachée (cf. exemples 26 et 31).

D'un point de vue sémantique, sérieusement et en serio admettent comme paraphrase les paraphrases par Ce que je fais sérieusement c'est te dire que $P$ (cf. exemple 39) ou Je suis sérieux en te disant que $P$ (cf. exemple 40) en français, ou Soy serio al decir que $P$ (cf. exemple 41) en espagnol :

(39) Ce que je fais sérieusement c'est te dire que je dois récupérer la quarantaine de Chinois qui se sont échappés dans votre casino.

(40) Je suis sérieux en te disant que je dois récupérer la quarantaine de Chinois qui se sont échappés dans votre casino.

(41) Soy serio al decir que mientras no cambies ni serás feliz ni conseguirás la paz que anhelas.

Récapitulons dans un tableau les emplois et les propriétés de sérieusement, seriamente et en serio que nous venons d'évoquer (cf. tableau 2). 
Tableau 2

\begin{tabular}{|c|c|c|c|c|c|c|c|c|c|}
\hline & \multicolumn{3}{|c|}{ SÉRIEUSEMENT } & \multicolumn{3}{|c|}{ SERIAMENTE } & \multicolumn{3}{|l|}{ EN SERIO } \\
\hline & sér $_{1}$ & $\operatorname{sér}_{2}$ & $\mathrm{sér}_{3}$ & $\operatorname{ser}_{1}$ & $\mathrm{ser}_{2}$ & $\operatorname{ser}_{x}$ & $e s_{1}$ & $e s_{x}$ & $e s_{2}$ \\
\hline Type d'Adv & $\begin{array}{l}\text { Adv de } \\
\text { manière } \\
\text { orienté } \\
\text { vers le } \\
\text { sujet }\end{array}$ & $\begin{array}{l}\text { Adv de } \\
\text { manière } \\
\text { quanti- } \\
\text { fieur in- } \\
\text { tensif }\end{array}$ & $\begin{array}{l}\text { Adv dis- } \\
\text { jonctif } \\
\text { de style }\end{array}$ & $\begin{array}{l}\text { Adv de } \\
\text { manière } \\
\text { orienté } \\
\text { vers le } \\
\text { sujet }\end{array}$ & $\begin{array}{l}\text { Adv de } \\
\text { manière } \\
\text { quanti- } \\
\text { fieur in- } \\
\text { tensif }\end{array}$ & $\begin{array}{l}\text { Adv dis- } \\
\text { jonctif } \\
\text { de style }\end{array}$ & $\begin{array}{l}\text { Adv de } \\
\text { manière } \\
\text { orienté } \\
\text { vers le } \\
\text { sujet }\end{array}$ & $\begin{array}{l}\text { Adv de } \\
\text { manière } \\
\text { quanti- } \\
\text { fieur in- } \\
\text { tensif }\end{array}$ & $\begin{array}{l}\text { Adv dis- } \\
\text { jonctif } \\
\text { de style }\end{array}$ \\
\hline $\begin{array}{l}\text { Existence } \\
\text { avérée? }\end{array}$ & $v$ & v & v & v & v & - & v & - & v \\
\hline Nombre & $\begin{array}{l}103 / \\
200\end{array}$ & $\begin{array}{l}43 / \\
200\end{array}$ & $\begin{array}{l}54 / \\
200\end{array}$ & $\begin{array}{l}156 / \\
200\end{array}$ & $\begin{array}{l}44 / \\
200 \\
\end{array}$ & - & $\begin{array}{l}300 / \\
328\end{array}$ & - & $\begin{array}{l}28 / \\
328 \\
\end{array}$ \\
\hline $\begin{array}{l}\text { Structure de } \\
\text { Surface }\end{array}$ & $S(X)$ & $S(X)$ & $S(X, Y)$ & $S(X)$ & $S(X)$ & - & $\mathrm{ES}(\mathrm{X})$ & - & $E S(X, Y)$ \\
\hline Clivée & v & - & - & - & - & - & - & - & - \\
\hline $\begin{array}{l}\text { Position initi- } \\
\text { ale détachée }\end{array}$ & - & - & v & - & - & - & - & - & $v$ \\
\hline Focus négation & $v$ & $\mathrm{v}$ & - & $\mathrm{v}$ & $\mathrm{v}$ & - & v & - & - \\
\hline $\begin{array}{l}\text { Réponse à lui } \\
\text { seul }\end{array}$ & $v$ & - & - & v & - & - & v & - & - \\
\hline $\begin{array}{l}\text { En tête de } \\
\text { n'importe quel } \\
\text { type de phrase }\end{array}$ & - & - & $v$ & - & - & - & - & - & $v$ \\
\hline $\begin{array}{l}\text { Coordonné } \\
\text { avec d'autres } \\
\text { Adv purement } \\
\text { d'intensité }\end{array}$ & $v$ & - & - & v & - & - & v & - & - \\
\hline $\begin{array}{l}\text { Position Adv } \\
\text { vis-à-vis de } \\
\text { l'Adj }\end{array}$ & $\begin{array}{l}\text { Post- } \\
\text { posé }\end{array}$ & $\begin{array}{l}\text { Anté- } \\
\text { posé }\end{array}$ & - & $\begin{array}{l}\text { Post- } \\
\text { posé }\end{array}$ & $\begin{array}{l}\text { Anté- } \\
\text { posé }\end{array}$ & - & $\begin{array}{l}\text { Post- } \\
\text { posé }\end{array}$ & - & - \\
\hline $\begin{array}{l}\text { Possibilité } \\
\text { de degré } \\
\text { (comparatif de } \\
\text { supériorité) }\end{array}$ & - & v & - & - & v & - & - & - & - \\
\hline $\begin{array}{l}\text { Possibilité de } \\
\text { surenchérisse- } \\
\text { ment }\end{array}$ & $v$ & v & - & $v$ & $v$ & - & $v$ & - & - \\
\hline
\end{tabular}


Tableau 2: (continu)

\begin{tabular}{|c|c|c|c|c|c|c|c|c|c|}
\hline & \multicolumn{3}{|c|}{ SÉRIEUSEMENT } & \multicolumn{3}{|c|}{ SERIAMENTE } & \multicolumn{3}{|l|}{ EN SERIO } \\
\hline & sér ${ }_{1}$ & sér $_{2}$ & $\operatorname{sér}_{3}$ & $\operatorname{ser}_{1}$ & $\mathrm{ser}_{2}$ & $\operatorname{ser}_{x}$ & $e s_{1}$ & $e s_{x}$ & $e s_{2}$ \\
\hline Type d’Adv & \begin{tabular}{|l|} 
Adv de \\
manière \\
orienté \\
vers le \\
sujet
\end{tabular} & \begin{tabular}{|l} 
Adv de \\
manière \\
quanti- \\
fieur in- \\
tensif
\end{tabular} & $\begin{array}{l}\text { Adv dis- } \\
\text { jonctif } \\
\text { de style }\end{array}$ & $\begin{array}{l}\text { Adv de } \\
\text { manière } \\
\text { orienté } \\
\text { vers le } \\
\text { sujet }\end{array}$ & $\begin{array}{l}\text { Adv de } \\
\text { manière } \\
\text { quanti- } \\
\text { fieur in- } \\
\text { tensif }\end{array}$ & $\begin{array}{l}\text { Adv dis- } \\
\text { jonctif } \\
\text { de style }\end{array}$ & \begin{tabular}{|l} 
Adv de \\
manière \\
orienté \\
vers le \\
sujet
\end{tabular} & $\begin{array}{l}\text { Adv de } \\
\text { manière } \\
\text { quanti- } \\
\text { fieur in- } \\
\text { tensif }\end{array}$ & $\begin{array}{l}\text { Adv dis- } \\
\text { jonctif } \\
\text { de style }\end{array}$ \\
\hline $\begin{array}{l}\text { Paraphrase de } \\
\text { (d'une) man- } \\
\text { ière / façon + } \\
\text { Adj } \\
\text { de manera / } \\
\text { forma + Adj }\end{array}$ & $v$ & - & - & $v$ & - & - & v & - & - \\
\hline $\begin{array}{l}\text { Paraphrase par } \\
\text { Le N est Adj / El } \\
N \text { es Adj }\end{array}$ & - & v & - & - & $v$ & - & - & - & - \\
\hline $\begin{array}{l}\text { Paraphrases } \\
\text { possibles : } \\
\text { Ce que je fais } \\
\text { Adv c'est te } \\
\text { dire que PJe } \\
\text { suis Adj en te } \\
\text { disant que } P \\
\text { Soy serio al } \\
\text { decir que } P\end{array}$ & - & - & v & - & - & - & - & - & $\mathrm{v}$ \\
\hline
\end{tabular}

\section{Polyfonctionnalité, grammaticalisation et subjectivation de sérieusement, seriamente et en serio}

Le fait qu'un même adverbe puisse remplir différentes fonctions et rentrer dans diverses sous-classes adverbiales a amené les linguistes à se poser la question de l'homonymie adverbiale : avons-nous affaire à une seule entité lexicale qui recouvre plusieurs emplois ? Ou plutôt à différentes unités homonymes ? À ce sujet nous adhérons aux postulats de Guimier (1996) et de Nølke (1990), entre autres. Nølke établit une nette distinction entre adverbe et complément adverbial ; c'est-à-dire, entre les éléments issus de la catégorie grammaticale adverbiale et les fonctions que ces adverbes peuvent remplir dans le discours, respecti- 
vement. Pour sa part, Guimier considère qu'estimer qu'il s'agit de plusieurs adverbes homonymes est contre-intuitif et ne répond pas à l'histoire du mot ; selon lui, il s'agit d'un seul et même adverbe qui connait des modes de fonctionnement différents (Guimier 1996, 3).

Nous nous sommes ainsi intéressée à l'histoire des adverbes sérieusement, seriamente et en serio. Bango de la Campa (2018) a étudié l'évolution historique des emplois de sérieusement et a constaté que les emplois comme adverbe de manière orienté vers le sujet sont les plus anciens en français : dans Frantext on en trouve des exemples dès le XVI ${ }^{\mathrm{e}}$ siècle (cf. exemple 42) - date assez tardive par rapport à celle d'autres adverbes en -ment, comme honnêtement et justement (déjà attestés au XII ${ }^{\mathrm{e}}$ siècle), apparemment (XIII ${ }^{\mathrm{e}}$ siècle), etc.

(42) Je le hay et fuy, de ce qu'il n'est pas assez jeu, et qu'il nous esbat trop serieusement, ayant honte d'y fournir l'attention qui suffiroit à quelque bonne chose (Michel de Montaigne, 1592, Essais).

Vient ensuite l'emploi comme adverbe de manière quantifieur intensif, attesté pour sérieusement au XVI ${ }^{\mathrm{e}}$ siècle (cf. exemple 43), et en dernier l'emploi comme adverbe disjonctif de style, datant de la fin du XVII ${ }^{\mathrm{e}}$ siècle (cf. exemple 44) :

(43) Cette volupté, pour estre plus gaillarde, nerveuse, robuste, virile, n'en est que plus serieusement voluptueuse. Et luy devions donner le nom du plaisir, plus favorable, plus doux et naturel : non celuy de la vigueur, duquel nous l'avons denommée (Michel de Montaigne, 1592, Essais).

(44) Car je ne voudrois pas jurer qu'elles ne parlassent sans langue. Je suis seûr du moins que si les paroles leur manquoient, elles auroient recours aux signes et aux gestes, pour faire entendre à tout le monde ce qu'elles ne pourroient dire. Serieusement elles ne peuvent se taire ; et deux ou trois exemples contraires sont des miracles qui ne font point de consequence (Dominique Bouhours, 1671, Les Entretiens d'Ariste et d'Eugène).

Nous nous sommes à notre tour appliquée à l'étude de l'évolution historique des emplois de seriamente et en serio. Le premier emploi attesté pour seriamente est celui en tant qu'adverbe de manière orienté vers le sujet $\left(\mathrm{XVII}^{\mathrm{e}}\right.$ siècle ; $\mathrm{cf}$. exemple 45) et le deuxième, l'emploi comme adverbe de manière quantifieur intensif (fin XVIII ${ }^{\mathrm{e}}$ siècle ; $\mathrm{cf}$. exemple 46) :

(45) como Rey catolico y piadoso sabiendo un tan graue daño desseó de remediarle en lo presente y ataxarlo en lo futuro, ordenó que el supremo Consejo de Inquisicion tratase con las veras que el caso pedia su remedio, a cuya causa este S. Tribunal hizo los dias passados publicar un edito, que remitió a todas las prouincias, en que debaxo de graues penas, excomunion, destierro, reclusion, y otras arbitrarias, segun la calidad de las culpas, manda seriamente que ningun Religioso diga ni escriua cosa alguna que pueda ser contra la estimacion de otro qualquiera Religioso o Religion, ni censure proposiciones de sus libros, o escritos (Anónimo, 1634, Carta de un caballero de Madrid). 
(46) Viéndole el lord tan seriamente desganado, acaba cuanto antes la cena. Los criados desocupan la mesa y desaparecen. El lord se levanta y toma otro asiento (Pedro Montengón, 1786, Eusebio).

Quant à en serio, le premier emploi attesté est celui en tant qu'adverbe de manière orienté vers le sujet (XVI ${ }^{\mathrm{e}}$ siècle ; $\mathrm{cf}$. exemple 47) et le deuxième, l'emploi comme adverbe disjonctif de style $\left(\mathrm{XX}^{\mathrm{e}}\right.$ siècle ; cf. exemple 48) :

(47) Mejor será con una vana, dos vacías, y dar una en el clavo y dos en la herradura y tres en el vacío, porque es cosa que repugna tratar de holgar y hablar en serio (Juan de Arce de Otárola, circa 1550, Coloquios de Palatino y Pinciano).

(48) Bueno, oiga usted, en serio... ¿ De verdad, de verdad que « esgorcia »? ii Gracias, señor Hernán !! (Se abrazan otra vez. Por el foro entra Oshidori) (Enrique Jardiel Poncela, 1932, Usted tiene ojos de mujer fatal).

Dans un souci de clarté, nous avons reflété les résultats obtenus sur l'évolution diachronique des emplois de sérieusement, seriamente et en serio dans le tableau suivant (cf. tableau 3) :

Tableau 3

\begin{tabular}{llll}
\hline Emploi / Adverbe & Sérieusement & seriamente & en serio \\
\hline Adverbe de manière orienté vers le sujet & 1592 & 1634 & circa 1550 \\
\hline Adverbe de manière quantifieur intensif & 1592 & 1786 & - \\
\hline Adverbe disjonctif de style & 1671 & - & début XXe siècle \\
\hline
\end{tabular}

Ces résultats viennent confirmer les hypothèses sur la diachronie des adverbes français et espagnols émises dans d'autres travaux. À propos des étapes de l'évolution diachronique de décidément, justement et apparemment et leurs équivalents en espagnol, Sonia Gómez-Jordana (2013) signale que les adverbes français ont connu une évolution un peu plus rapide que les adverbes espagnols. Jusqu'en français préclassique les adverbes français ne possèdent qu'une valeur en tant qu'adverbe intégré à la proposition, et notamment en tant qu'adverbe de manière. Les emplois comme adverbe de phrase n'apparaissent qu'à partir du début du XVII ${ }^{\mathrm{e}}$ siècle et ce ne sera qu'au XVIII ${ }^{\mathrm{e}}$ siècle que cette valeur se consolidera. Pour ce qui est de l'espagnol, la seule valeur existante jusqu'au XVII ${ }^{\mathrm{e}}$ siècle sera la valeur en tant qu'adverbe de manière et l'adverbe d'énonciation n'apparaitra qu'au XVIII ${ }^{\text {e }}$ siècle, et ceci seulement pour certains adverbes.

D'un point de vue diachronique les adverbes contemporains en -ment sont le résultat d'un processus de grammaticalisation (ou, si l'on préfère, d'une gramma- 
ticalisation au sens étroit et d'un processus de subjectivation). Elizabeth Traugott définit la grammaticalisation comme suit : « Grammaticalization [...] is that subset of linguistic changes whereby lexical material in highly constrained pragmatic and morphosyntactic contextes becomes grammatical, and grammatical material becomes more grammatical » (Traugott 1996, 183).

En effet, les adverbes en -ment n'existaient pas en latin mais sont d'origine romane. En proto-roman il s'est produit la coalescence (Lehman 1995) du substantif féminin mens, mentis (au sens de l'esprit, l'intention) et de l'adjectif féminin (par exemple, clara), ce qui a donné naissance à une nouvelle forme adverbiale : clara mente, qui avait le sens de 'dans une intention claire' (Combettes 2008). Celui-ci constitue un bon exemple de grammaticalisation au sens étroit, puisqu'il remplit toutes les conditions établies par les spécialistes en la matière pour décrire ce phénomène : a) il y a changement de catégorie (recatégorisation ou dégradation, dans le sens de Meillet 1912) : avec la formation de l'adverbe en -ment nous « descendons » de la catégorie nominale et la catégorie adjectivale à la catégorie adverbiale ; b) il y a désémantisation (ou affaiblissement du sens) du substantif latin mens, mentis, qui perd sa signification initiale d'esprit ou intention ; c) il y a perte d'autonomie du substantif latin, qui ne pourra plus fonctionner tout seul ; et enfin d) il y a perte phonétique, puisque le [t] et la voyelle finale [e] du substantif mente ne se prononcent plus actuellement en langue française.

Une fois les adverbes en -ment intégrés au système des langues romanes, leur évolution ne s'est toutefois pas arrêtée ; au contraire, elle a continué, mais cette fois-ci sur le plan sémantique : les changements qui s'en suivront seront de cette nature. Ces changements sémantiques constituent un clair exemple de ce qu'on appelle grammaticalisation au sens large ou, si l'on préfère, subjectivation. Traugott propose la definition suivante de subjectivation : «subjectification» refers to a pragmatic-semantic process whereby «meanings become increasingly based in the speaker's subjective belief state / attitude toward the proposition>, in other words, towards what the speaker is talking about " (Traugott 1995, 31). Comme le signale Combettes $(2008,140)$, grâce au processus de subjectivation, le sens de l'expression évolue en passant du monde de la référence au monde du locuteur ; c'est-à-dire à la propre subjectivité de celui-ci vis-à-vis du contenu de sa proposition.

Notre hypothèse concernant la diachronie de ces adverbes est que l'adverbe de manière orienté vers le sujet a donné lieu à l'adverbe de manière quantifieur intensif et, plus tard, à l'adverbe disjonctif de style (ou adverbe d'énonciation) à la suite d'un processus de subjectivation. Les dates d'émergence des différents emplois des trois adverbes étudiés ainsi le corroborent. Il existe par ailleurs un autre paramètre important à être pris en compte : la place de l'adverbe vis-à-vis de l'élément sur lequel il porte. Nous avons constaté que le déplacement de 
l'adverbe vers la gauche de l'énoncé infléchit son interprétation vers l'intensif, vers le subjectif : l'adverbe de manière orienté vers le sujet devient, antéposé, adverbe de manière quantifieur intensif (cf. Flament-Boistrancourt 2011). De même, Schlyter $(1977,43)$ fait observer que « les adverbes émotifs et non-focus ont tendance à figurer aussi loin à gauche que possible » et que « les adverbes qui figurent surtout en position initiale [...] ne peuvent généralement pas être focus, mais sont souvent liés au locuteur » (ib.). Comme nous l'avons vu, la place prototypique des adverbes d'énonciation est en tête de phrase et en position détachée. Il semblerait donc que le processus de subjectivation des adverbes français et espagnols soit étroitement lié à leur place dans l'énoncé : plus un adverbe se trouvera à gauche de l'énoncé, plus nous aurons affaire à un élément lié au locuteur et à sa subjectivité.

Voilà pourquoi nous pouvons affirmer que certains adverbes contemporains en -ment (et en particulier, sérieusement et seriamente) sont le résultat d'un processus de grammaticalisation au sens étroit et d'un processus de grammaticalisation au sens large.

\section{Conclusion}

Dans cet étude nous avons voulu identifier et justifier par des propriétés linguistiques objectivement repérables les différents emplois de l'adverbe français sérieusement et des adverbes espagnols seriamente et en serio ; à savoir adverbe de manière orienté vers le sujet et adverbe d'énonciation (sérieusement, seriamente et en serio), adverbe de manière quantifieur intensif (sérieusement et seriamente) et adverbe disjonctif de style - ou d'énonciation - (sérieusement et en serio).

Les recherches menées sur la diachronie des deux langues romanes montrent (français et espagnol) que l'emploi adverbial le plus ancien est celui en tant qu'adverbe intégré à la proposition et, plus précisément, comme adverbe de manière orienté vers le sujet (cf. sérieusement $t_{1}$ et seriamente ${ }_{1}$, mais aussi en serio $_{1}$ ). À son tour, l'emploi en tant qu'adverbe de manière quantifieur intensif semble être apparu à une époque assez précoce de l'histoire de la langue française (seriamente ${ }_{2}$ ), beaucoup plus tardive pour la langue espagnole (seriamente $_{2}$ ). Enfin, les emplois comme adverbe de phrase viennent toujours en dernière position dans l'évolution interne de chaque adverbe (sérieusement ${ }_{3}$ et en serio $_{2}$ ).

Le passage d'un emploi adverbial à un autre pourrait s'expliquer à l'aide des notions de grammaticalisation et de subjectivation (dans le sens de Traugott), c'est-à-dire, comme l'aboutissement d'un processus de grammaticalisation au sens étroit et d'un processus de grammaticalisation au sens large. Dans certains 
cas les trois emplois coexisteront dans la langue contemporaine (cf. sérieusement en français) ; dans d'autres, l'emploi en tant qu'adverbe de manière quantifieur intensif aura à ce jour disparu (i.e. en serio en espagnol) ; et dans d'autres cas encore, ce sera l'adverbe disjonctif de style qui se sera effacé dans la langue contemporaine (i.e. seriamente).

\section{Références bibliographiques}

Álvarez-Prendes, Emma, Différents types d'emplois et propriétés de l'adverbe « sérieusement » en français contemporain, Scolia 30 (2016), 89-104.

Álvarez-Prendes, Emma, Les adverbes « honnêtement » et « honestamente » : emplois, propriétés et subjectivation, soumis.

Anscombre, Jean-Claude/Donaire, María Luisa/Haillet, Pierre Patrick (edd.), Opérateurs discursifs du français. Éléments de description sémantique et pragmatique, Bern, Lang, 2013.

Anscombre, Jean-Claude, et al., Indices et attitudes énonciatives : le cas d' " apparemment ", Langue française 161 (2009), 39-58.

Bango de la Campa, Flor, Diachronie des opérateurs sémantico-pragmatiques, in : Anscombre, Jean-Claude/Donaire, Maria Luisa/Haillet, Pierre Patrick (edd.), Opérateurs discursifs du français 2, Bern, Lang, 2018, 421-480.

Combettes, Bernard, Théories du changement et variations linguistiques : la grammaticalisation, Pratiques. Linguistique, littérature, didactique 137-138 (2008), 13-145.

Flament-Boistrancourt, Danièle, « Sinon » vs. « autrement » : si proches, si loin..., Langages 184 (2011), 129-146.

Greenbaum, Sidney, Studies in English Adverbial Usage, London, Longman, 1970.

Gómez Jordana Ferary, Sonia, Les étapes du français et de l'espagnol à la lumière de la diachronie des marqueurs : une évolution en cadence ?, Revue de Sémantique et Pragmatique 33-34 (2013), 177-194.

Guimier, Claude, Les adverbes du français. Le cas des adverbes en -ment, Paris, Ophrys, 1996.

Lehman, Christian, Thoughts on Grammaticalization, München/Newcastle, Lincom Europa, 1995.

Meillet, Antoine, L'évolution des formes grammaticales, in : id., Linguistique historique et linguistique générale, Paris, Champion, 1912, 131-148.

Molinier, Christian, Une classification des adverbes en -ment, Langue française 88 (1990), 28-40.

Molinier, Christian, Les adverbes d'énonciation. Comment les définir et les sous-classifier, Langue française 161 (2009), 9-21.

Molinier, Christian/Levrier, Françoise, Grammaire des adverbes. Description des formes en -ment, Genève, Droz, 2000.

Nølke, Henning, Les adverbiaux contextuels : problèmes de classification, Langue française 88 (1990), 12-27.

Rodríguez Ramalle, María Teresa, La gramática de los adverbios en -mente o cómo expresar maneras, opiniones y actitudes a través de la lengua, Madrid, UAM Ediciones, 2004.

Schlyter, Suzanne, La place des adverbes en -ment en français, thèse de doctorat, Konstanz, Universität Konstanz, 1977. 
Traugott, Elizabeth Closs, Subjectification in grammaticalisation, in : Stein, Dieter/Wright, Susan (edd.), Subjectivity and Subjectivisation, Cambridge, Cambridge University Press, 1995, 31-54.

Traugott, Elizabeth Closs, Grammaticalization and lexicalization, in : Brown, Katherine/Miller, Jim (edd.), Concise Encyclopedia of Syntactic Theories, Oxford, Pergamon, 1996, 181-187. 